\title{
Planejamento Estratégico Situacional em Saúde: Um Desafio no Gerenciamento de uma Unidade Básica de Saúde em Paulo Afonso - BA
}

Damaris Lopes de Martins Silva ; Sâmela dos Santos Vieira ${ }^{2}$

Resumo: Sendo o Planejamento Estratégico como importante recurso para otimização e melhoria das ações de saúde, especialmente na Estratégia Saúde da Família, este estudo exploratório descritivo, do tipo relato de experiência, teve como objetivo analisar a utilização do Planejamento Estratégico em uma unidade de Saúde da Família, na cidade de Paulo Afonso - BA, no que se refere à saúde do adolescente. Os resultados foram três situações-problema: 1. Falta de interação e comunicação nas relações nas equipes da unidade de saúde; 2 . Recursos humanos insuficientes para atender a demanda de saúde; e 3. Inexistência de ações e incentivos para a promoção da saúde do adolescente. O que se pode perceber é uma fragilidade na organização das demandas de saúde, o que acaba por se traduzir na dificuldade de gestão das equipes de saúde com fins estratégicos. Neste sentido, conclui-se que uma das ferramentas para traçar os nós críticos das situações problema é o Planejamento Estratégico, pois possibilita um levantamento das causas e acrescenta valores a pratica gerencial, oferecendo suporte para melhoria das ações implementadas.

Palavras-chave: Programa de Saúde da Família. Gestão em Saúde. Planejamento Estratégico.

\section{Strategic Planning in Situational Health: A Challenge in The Management of Unit of Basic Health in The City of Paulo Afonso - BA}

\begin{abstract}
Being Strategic Planning as an important resource for optimization and improvement of health actions, especially in the Family Health Strategy, this exploratory-descriptive study, the type experience report, aimed to analyze the use of strategic planning in a Health Unit Family, in Paulo Afonso - BA, with regard to adolescent health. The results were three problem situations: 1. Lack of interaction and communication in relationships in health unit teams; 2. Insufficient human resources to meet the health demands; and 3. Lack of actions and incentives for promoting adolescent health. What can be seen is a weakness in the organization of health demands, which finally results in the difficulty of managing health teams with strategic purposes. In this sense, it is concluded that one of the tools to trace the critical nodes of problem situations is the Strategic Plan, as it allows an assessment of the causes and adds value to management practices, providing support for improvement of the actions implemented.
\end{abstract}

Keywords : Health Family Program . Health Management. Strategic Planning.

\footnotetext{
${ }^{1}$ Especialização (2013), pelo Programa Nacional de Formação em Administração Pública - Pós-Graduação em Gestão em Saúde (PNAP) na Universidade Federal do Vale de São Francisco. Graduada em Letras - Português e Inglês(Licenciatura) pela Faculdade Sete de Setembro Fasete (2007). Graduanda do VII período de Enfermagem pela Faculdade Sete de Setembro Fasete. E-mail: damarislopes@ yahoo.com.br ${ }^{2}$ Professora Assistente no Colegiado de Psicologia, na Universidade Federal do Vale do São Francisco - UNIVASF. Doutoranda e Mestre em Psicologia pela Universidade Federal de Pernambuco - UFPE, na linha Processos Psicossociais, Poder e Práticas Coletivas. E-mail: samella.vieira@hotmail.com
} 


\section{Introdução}

A pesquisa sobre o tema foi influenciada pelas inquietações surgidas nas experiências vivenciadas na formação acadêmica em Enfermagem, a partir da oportunidade de realizar estágios curriculares na Atenção Básica. No curso de especialização, ampliou-se o interesse por esta temática da Saúde Coletiva e sua interface com a gestão, especialmente quando, na disciplina de Planejamento Estratégico Governamental (PEG), utilizou-se a metodologia de diagnostico de situações-problema na Unidade de Atenção Básica da Saúde da Família (UBSF). Diante destas experiências teóricas e práticas, foi se definindo o desejo de estudar na Especialização de Gestão em Saúde os desafios para a efetivação do Planejamento Estratégico em Saúde no contexto de Atenção Primária à Saúde.

Para que possamos avançar nas discussões, é importante a definição de Planejamento Estratégico Situacional (PES) proposto por Matus (1993). Para o autor (1989; 1993), planejar é como preparar-se para a ação. Segundo Mintzberg (2003), "planejar é articular, coordenar atividades e resultados" (p. 32). O PES se trata de um método de planejamento por problemas, e evidencia os problemas mais complexos e que não há solução normativa. Os problemas são abordados em dimensões política, econômica, social, e muitas vezes culturais. Assim, as soluções em sua grande maioria dependem exclusivamente de recursos de outras esferas e da interação dos atores envolvidos.

O PES possui quatro momentos para o processamento técnico-político dos problemas: 1. O momento explicativo ocorre a seleção e a análise do problema; 2. O momento normativo reconhece os nós críticos e são elaboradas propostas de ação, com a construção de planilhas de programação de ações; 3. O momento estratégico onde as ideais são construídas para viabilizar o plano ( nesta etapa o ator não controla todos os recursos para sua execução, exigindo assim cooperação de outros atores e da equipe); e 4. O momento tático-operacional é a gestão do plano, as pessoas assumem compromissos para executar tarefas específicas, em tempos bem definidos (ARTMANN, 1997).

A ideia de planejamento estratégico, ganha duas configurações, ao observamos as acepções para cada palavra. Por planejamento, o mesmo figura como uma proposta contínua de ações, e tem como perspectiva fundamental alcançar o que foi planejado, independentemente dos prazos colocados; o que permite que possamos entender que o ato de planejar possui uma visão de futuro. Já a noção de estratégia possui a visão no presente, antecipando os possíveis obstáculos que poderão surgir na situação-problema, possibilitando sair da mesma no contexto presente.

Matus (1993) aponta que a utilização de tal proposta no campo da saúde vem sendo uma crescente, pois permite o planejamento dos serviços de saúde. Se configura como interessante ferramenta que deve ser incorporada pela equipe que trabalha nas unidades de Saúde da Família e deve ser incorporado no gerenciamento dos serviços de Saúde (CIAMPONE, 2001).

No que concerne ao campo da saúde pública, o PES é uma ferramenta reconhecida e indicada pelo Ministério da Saúde. Segundo Pereira (2011), para que se possa ter efetividade no campo da 
gestão, faz-se necessário que a gerência de tais UBSF possuam gestores preparados, na medida em que o trabalho de gestão exige complexidade gerencial e onde os problemas demandados pressupõem que o gestor, no desempenho de suas funções, dimensione as necessidades interna da UBSF. Para isso, o mesmo deve traçar metas, estratégias de resolução dos conflitos e, nessa égide, planos de ação a partir do diagnóstico situacional.

O autor supracitado sabiamente coloca que as etapas de acompanhamento e avaliação sejam seguidas de forma criteriosa, para que se possa identificar as ações que estão ou não surtindo efeito. Uma vez que o planejamento estratégico é uma das ferramentas muito utilizadas nas gerencias das organizações, por se tratar de um processo formalizado para gerar resultados a partir da sistematização das decisões (PEREIRA, 2011), sua importância também nas organizações de saúde pública é salutar.

Existem grandes barreiras no processo de planejamento estratégico em saúde, dos quais chama atenção a imensa rotatividade de profissionais e a incipiência numa política de planejamento de fato efetiva. Assim, é possível destacar que os profissionais de saúde muitas vezes não estão abertos à proposta de planejamento. Entretanto, é sabido que as UBSF se configuram como ambientes que exigem constante reflexão da práxis. Assim sendo, o Planejamento Estratégico contribui para o alcance de metas, soluções de problemas e necessidades de saúde e dos serviços oferecidos à comunidade. Há, portanto, uma necessidade diária de avaliação desses serviços para que haja um bom desempenho gerencial da unidade de saúde.

Considerando que as UBSF é a porta de entrada prioritária para os níveis de atenção à saúde, quando bem estruturados atendem as necessidades de saúde da comunidade com boa resolutividade. É de suma importância que haja a participação da toda a equipe da unidade em todas as etapas do planejamento, no levantamento dos problemas, no plano de ação e no acompanhamento.

Para desenvolver um planejamento estratégico, é necessário conhecer a realidade, as rotinas e equipe da unidade básica de saúde, a organização de saúde como um todo, assim, a formulação, priorização e explicação de problemas de saúde da população serão visualizados, permitindo assim ações de saúde mais focais e efetivas. Segundo Mendes (1994), o Planejamento em Saúde é uma ação que gera transformação, de uma situação inaceitável para outra desejável e ideal, sendo um aliado para o gestor e sua equipe, pois o planejamento em saúde possui ferramentas importantes para identificar problemas.

De acordo com Cecílio (1997), o sucesso na execução do plano exige precisão e comunicação, com definição das responsabilidades, monitoramento e avaliação. Há necessidade de alguns rearranjos institucionais, envolvimento de gerências formais com as operações. No método do PES, planejamento e gestão são inseparáveis.

De acordo com Matus (1993), para iniciar o ato de planejar, necessita-se saber muito bem quais os problemas que incomodam e se os mesmos importunam a mais alguém. Para eleger o 
problema é necessário considerar as seguintes questões: Achamos que o problema pode ter solução? Ainda não sabemos qual é a solução? Achamos que agora é um bom momento para atuar sobre esse problema?

Partindo dos questionamentos acima, o presente relato de experiência teve como objetivo analisar a utilização do Planejamento Estratégico no contexto de uma UBSF na cidade de Paulo Afonso - BA. A guisa de exemplificação, foi contemplada a demanda de saúde do adolescente, que será melhor delineada na seção de resultados do estudo.

\section{Método}

O estudo se enquadra na perspectiva da pesquisa em qualitativa em Saúde. A pesquisa qualitativa trabalha com um universo de significados, motivos, aspirações, crenças, valores e atitudes, o que corresponde a um espaço mais profundo das relações, dos processos e dos fenômenos que não podem ser reduzidos à operacionalização de variáveis (MINAYO, 1993). Sendo a pesquisa de cunho exploratório-descritivo, se baseia no levantamento do perfil das demandas de saúde de adolescentes, buscando de modo detalhado o conhecimento sobre este grupo especifico, tentando elencar as problemáticas que envolvem questões no atendimento à saúde do adolescente.

A UBSF que foi contemplada pela experiência possui uma equipe de saúde da família, composta por um médico, um dentista, uma enfermeira, uma auxiliar de enfermagem e agentes comunitários de saúde (ACS), recepcionistas e serviços gerais. O trabalho aqui abordado está centrado no Planejamento Estratégico-Situacional de Carlos Matus, aportada também por Rivera (1995), mas, as duas fortes vertentes do PES tem seu expoente maior em Carlos Matus, e o pensamento estratégico, desenvolvido por Mário Testa.

Soma-se a este enfoque, a compreensão relativa às particularidades das organizações de saúde, enquanto processo produtivo e de divisão de poder (Mintzberg, 1989) e suas consequências para a prática de planejamento e processos de mudança gerencial.

\section{Resultados e Discussão}

Os resultados serão dispostos a partir de diagnose situacional da problemática do foco de estudo. A partir das experiências em campo e em contato com os profissionais de saúde da UBSF, foi possível identificar o número crescente de gravidez na adolescência. No que se refere ao planejamento familiar, as doenças sexualmente transmissíveis e ao uso de métodos contraceptivos, se configuram como as maiores demandas de grande parte das adolescentes nesta UBS. De acordo com o que foi 
observado, as meninas iniciam a vida sexual ativa muito cedo, muitas delas ainda na puberdade. Como este assunto ainda é considerado tabu, não contam para o agente comunitário de saúde (ACS) nem para outros profissionais sobre a vida sexual, na maioria das vezes por medo da revelação para seus pais, o que torna difícil estimar qual o número real de adolescentes com tais problemáticas na comunidade, bem como traçar estratégias de enfrentamento baseadas nas ações de educação em saúde.

A partir do exposto, no processo de avaliação do diagnóstico na unidade básica de saúde em estudo, foram identificados os seguintes problemas destacados a seguir. Os mesmos foram elencados a partir da criação de figuras que fazem parte do modus operandi da PES, onde é possível visualizar, além da situação-problema, suas causas e consequências.

Figura 1: Falta de comunicação e interação nas relações entre as equipes da UBSF

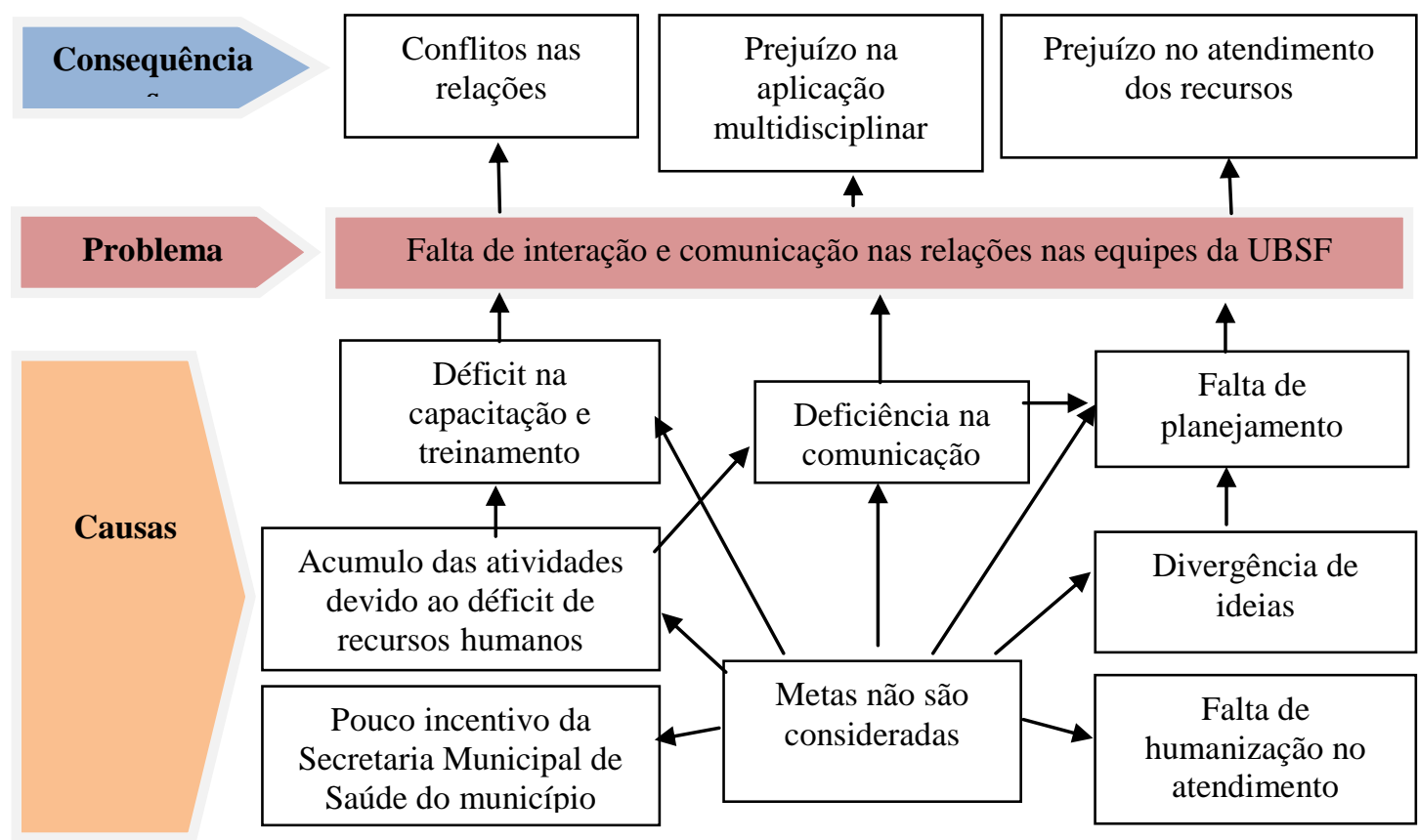

Cada uma dessas complexidades apresentadas na figura 1 são implicações operacionais distintas sobre a capacidade de compreensão das necessidades de saúde, bem como de cumprimento dos princípios de equidade, integralidade e universalidade das ações estabelecidas pelo SUS. A figura traz dados importantes, porque de maneira ampliada, sinaliza a falta de interação e comunicação das relações dos profissionais que compõem as equipes das UBSF. Isso posto porque, em relação as causas, é possível perceber que há uma falta de planejamento que de fato dê conta de algumas deficiências tanto na comunicação, quanto de capacitação e treinamento para os profissionais. Uma vez que há pouco incentivo pela Secretaria Municipal de Saúde, segundo relatam os profissionais, a noção de metas não são consideradas, o que torna excludente ações que se baseiem num atendimento resolutivo e de qualidade. A falta de humanização, desse modo, traz como consequências diretas o 
conflito nas relações dos membros do grupo, além de prejuízos no atendimento dos recursos e num trabalho multidisciplinar; essa problemática interfere sobremaneira em ações à saúde de adolescentes e jovens.

Figura 2: Recursos Humanos insuficientes para atender a demanda.



A figura 2, apresenta como problemática central os prejuízos causados pela insuficiência de recursos humanos na UBSF, implicando em um atendimento deficiente aos usuários daquela comunidade, e sobrecarga de trabalho da equipe de saúde. Tal déficit de recursos humanos exige da equipe esforços para atender a demanda espontânea e outras ações da atenção primária. Feuerwerker (2007), diz que "as equipes enfrentam muitas dificuldades para lidarem com a demanda espontânea e a atenção a elas quase sempre se conforma no esquema queixa-conduta". O crescimento demográfico na área de abrangência da UBSF inviabiliza um atendimento adequado pela insuficiência de profissionais. Para o usuário, os prejuízos são muitos, como por exemplo, o tempo de espera para ser atendido é muito maior do que o realmente necessário. A consequência da falta de recursos humanos inviabiliza o acolhimento como uma estratégia do modelo assistencial, premissas básicas do Programa Saúde da Família. 
Figura 3: Inexistência de ações e incentivos para a promoção da saúde do adolescente.

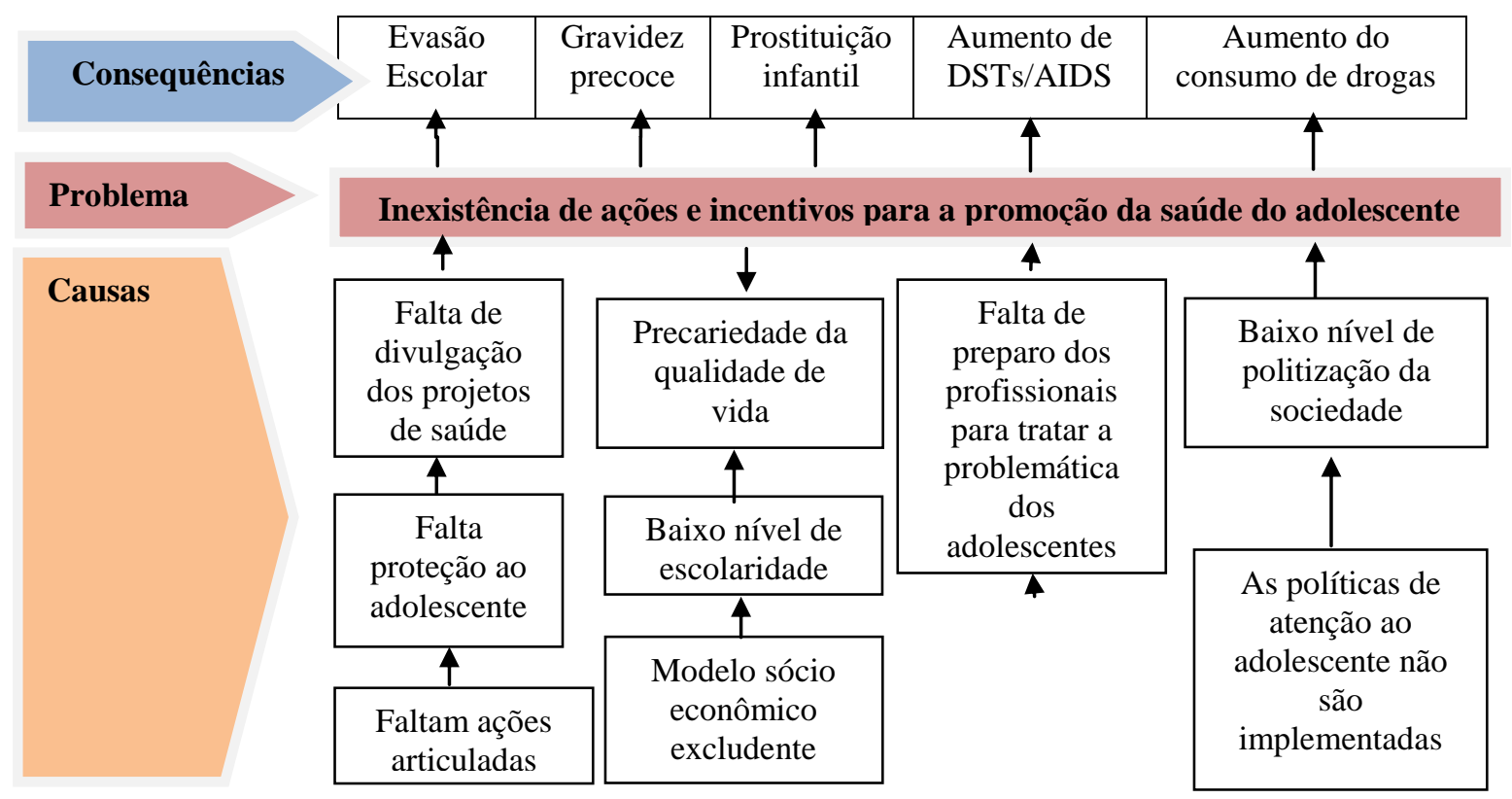

As duas situações-problema supracitadas, dão por sua vez margem para a problemática no que se refere ao atendimento na promoção da saúde do adolescente. Queiroz (2010) afirma que há um despreparo dos profissionais e dos serviços de saúde em relação ao cuidado com a saúde dos adolescentes, no sentido de atender as peculiaridades deste grupo, faltando suporte apropriado para as demandas. As ações requerem que os mesmos sejam orientandos, prevenindo ou recuperando a saúde do adolescente, e as UBSF devem ser um espaço de confiança, onde os profissionais são preparados para realizarem educação em saúde.

Ferrari (2006) orienta que a captação dos adolescentes deve ser realizada por meio de ações estratégicas, tanto nas unidades de saúde quanto na comunidade. É importante, no entanto, o profissional de saúde crie oportunidades de contato com os adolescentes.

Observa-se na figura 3, que as ações para promoção da saúde do adolescente são falhas, colocando-os em vulnerabilidade, a exemplo da gravidez e exposição ao abuso às drogas. É possível perceber que as maiores preocupações existentes na promoção da saúde do adolescente é apenas no tocante às questões relativas a sexualidade, sobretudo no caso da mulher pra evitar a gravidez não planejada.

A pouca resolutividade nas ações direcionadas ao cuidado integral do adolescente, pode ser visualizado na não implementação de políticas de atenção ao adolescente. A falta de preparo dos profissionais para atender as demandas desse público pode ser vista na pouca ou nenhuma articulação intersetorial entre serviços de saúde/educação que integrem, em sua complexidade, tal grupo. 
Diante do exposto, há necessidade dos profissionais serem mais bem preparados e resolutivos, pois a abordagem aos adolescentes certamente requer todas essas qualidades profissionais. É indispensável que a equipe de saúde seja comprometida para que a prevenção de problemas que geram as necessidades de saúde nesta fase, visando reduzir riscos e promover a saúde e a qualidade de vida do adolescente.

Percebe-se que a UBSF estudada não possui um planejamento, o sucesso do planejamento estratégico, depende da efetivação dos resultados. Embora o gestor da unidade em questão utilize dados epidemiológicos como fonte de informação que identifica as necessidades de saúde da sua área de abrangência, mas os dados epidemiológicos por si só não resultam em uma ação efetiva para a solução dos problemas identificados. Assim, o planejamento estratégico em saúde possui uma visão de transformação social dos dados epidemiológicos apresentados, intervindo na formulação de uma nova realidade social.

\section{Considerações Finais}

$\mathrm{Na}$ prática gerencial dos gestores e gerentes da UBSF pesquisada, foi evidenciado sobremaneira a perda de controle no alcance das metas e a ausência de plano de ação que alcance resultados. Sabe-se que a pratica gerencial envolve desafios, mas que é possível obter resultados satisfatórios através do planejamento estratégico e de profissionais qualificados para atuarem na unidade básica de saúde. O planejamento estratégico auxilia, no desenvolvimento da assistência em saúde e para o redirecionamento das ações a partir dos fatores intervenientes identificados.

Os desafios enfrentados no cotidiano para a efetiva na implementação do planejamento estão associados a fatores internos e externos, é importante que exista uma política para o planejamento em saúde institucionalizada, bem como profissionais qualificados para atuarem no gerenciamento de unidades básicas de saúde.

Há algumas questões complexas na situação problema da UBSF do município em questão. É importante dizer que as diferentes formas de representação e compreensão dos problemas de saúde pelos diversos grupos sociais envolvidos, incluindo a população e a complexidade dos fenômenos que caracterizam a realidade e a intervenção. A existência de problemas não significa ausência de soluções; o conhecimento, interesse e a capacidade de agir do ator social sobre a situação problema, vão influenciar sua percepção sobre o problema de saúde e propiciar a busca pela resolutividade da mesma. 
Referências

ARTMANN E.; AZEVEDO C. S.; SÁ M. C. Possibilidades de aplicação do enfoque estratégico de planejamento no nível local de saúde: análise comparada de duas experiências. Caderno Saúde Pública, Rio de Janeiro, 1997.

AZEVEDO, C. S. Gestão hospitalar: a visão dos diretores de hospitais públicos do Município do Rio de Janeiro. Revista de Administração Pública, vol. 29, n. 3, p. 38-37, 1995.

BRASIL. Ministério da Saúde. Secretaria de Atenção à Saúde. Departamento de Ações Programáticas Estratégicas. Marco teórico e referencial: saúde sexual e saúde reprodutiva de adolescentes e jovens [Internet] Brasília(DF):Ministério da Saúde,2006. citado 2010 Jan 15 Disponível em: http://bvsms.saude.gov.br/bvs/publicacoes/07_0471_M.pdf

CIAMPONE MHT, Peduzzi M. Planejamento Estratégico como instrumento de gestão e assistência. In: Brasil. Instituto para o Desenvolvimento da Saúde. Universidade de São Paulo. Ministério da Saúde. Manual de Enfermagem: Programa Saúde da Família. São Paulo; 2001.

FERRARI RAP, Thomson Z, Melchior R. Atenção à saúde dos adolescentes: percepção dos médicos e enfermeiros das equipes da saúde da família. Cad. Saúde Pública, v.22, n. 11, p. 2491-2493, nov. 2006.

FEUERWERKER, Laura. Modelos Tecnoasssistenciais, Gestão e Organização do Trabalho em Saúde: nada é indiferente no processo de luta para a consolidação do SUS. Temporalis, São Luis: Associação Brasileira de Ensino e Pesquisa em Serviço Social, 2007.

MATUS, C. Fundamentos da planificação situacional. In: RIVERA, F.J.U. (Org.). Planejamento e programação em saúde: um enfoque estratégico. São Paulo: Cortez, 1989.

MATUS, C. Política, planejamento e governo. Brasília: IPEA, 1993. MENDES, E. V. Planejamento e programação local da vigilância da saúde no distrito sanitário. Brasília: OPAS/OMS, 1994 MINAYO, M. C. S. Pesquisa social. Rio de Janeiro: Vozes, 1993.

MINTZBERG, Henry et.al. O processo da estratégia: conceitos, contextos e casos selecionados. 4. ed. Porto Alegre: Bookman, 2003.

MINTZBERG, Henry. Criando organizações eficazes: estruturas em cinco configurações. São Paulo: Atlas, 1995

PEREIRA, M. F. Administração estratégica da saúde. Florianópolis: Fundação Boiteux; Departamento de Ciências da Administração. UFSC, 2011.

QUEIROZ MVO, Ribeiro EMV, Pennafort VPS. Assistência ao adolescente em um serviço terciário: acesso, acolhimento e satisfação na produção do cuidado. Ver. Texto Contexto Enfermagem. 2010.

RIVERA, U. Planejamento e programação em saúde: um enfoque estratégico. São Paulo: Cortez, $3^{\mathrm{a}}$ ed. p. 222, 1989.

\section{Como citar este artigo (Formato ABNT):}

SILVA, D.L.M.; VIEIRA, S.S. Planejamento Estratégico Situacional em Saúde: Um desafio no Gerenciamento de uma Unidade Básica de saúde em Paulo Afonso - BA. Id on Line Revista de Psicologia, Abril de 2015, vol.9, n.26, Supl. Esp. p. 109-117. ISSN 1981-1189.

Recebido: 05/03/2015

Aceito:08/03/2015 